\title{
Molecular and Probiotic Characterizations of Lactobacillus reuteri DSM 12246 and Impact of pH on Biomass and Metabolic Profile in Batch-Culture
}

\author{
Mohamed Gamal El-Ziney \\ Lab of Functional Foods, Department of Dairy Sciences and Technology, Faculty of Agriculture, El Shatby Campus, \\ Alexandria University, Alexandria, Egypt \\ Email: elziney@yahoo.com
}

How to cite this paper: El-Ziney, M.G. (2018) Molecular and Probiotic Characterizations of Lactobacillus reuteri DSM 12246 and Impact of $\mathrm{pH}$ on Biomass and Metabolic Profile in Batch-Culture. Advances in Microbiology, 8, 18-30. https://doi.org/10.4236/aim.2018.81002

Received: December 10, 2017 Accepted: January 12, 2018

Published: January 16, 2018

Copyright $\odot 2018$ by author and Scientific Research Publishing Inc. This work is licensed under the Creative Commons Attribution International License (CC BY 4.0).

http://creativecommons.org/licenses/by/4.0/

\begin{abstract}
Lactobacillus reuteri is a powerful probiotic and adjunct functional culture candidate received a lot of scientific attention as it is one of the few endogenous "Lactobacillus" species found in the gastrointestinal tract of vertebrates, including humans, rats, pigs and chicken. The organism has been widely utilized as a probiotic in humans and animals for many years. In the present work L. reuteri DSM 12243; a high reuteri producer strain was molecularly characterized by $16 \mathrm{SrRNA}$ and RAPD analyses further, the probiotic properties including acid resistance, bile tolerance, adhesion to epithelial gastric cells, and antibiotic susceptibility were also assessed. Furthermore, the effect of $\mathrm{pH}$ on biomass production and metabolic profile of L. reuteri DSM 12246 in batch-culture was studied. The L. reuteri DSM 12246 showed a high similarity with L. reuteri strain I49 KR 36477 (100\%) and type strain ATCC 55730 (99\% of identity). The strain adhered well to $\mathrm{CaCO}_{2}$ cells and showed to be a highly resistance to acid juice ( $\mathrm{pH} 3.0$ ), with $0.7 \log _{10} \mathrm{cfu} / \mathrm{ml}$ reduction in count after $60 \mathrm{~min}$ exposition. There is no significant change in the cell count after exposure to bile salts. In batch-cultures, at low $\mathrm{pH}$ values both glucose consumption and metabolites were low while the production of lactic acid was noticeable. Maximum biomass was reached at $\mathrm{pH} 5.5$, with growth rate of $\mu=$ $0.641 / \mathrm{h}$. The switch in $\mathrm{pH}$ values from 3.7 to 6.7 resulted in raising of glucose depletion as well as in the yield of acetate and ethanol. It is concluded that $L$. reuteri DSM 12246 was deemed as a successful candidate to be used as potential probiotic.
\end{abstract}

\section{Keywords}

Lactobacillus reuteri DSM 12246, Probiotic, Acid and Bile Tolerance, Adherence, $\mathrm{pH}$, Metabolic Profile 


\section{Introduction}

Since Gerhard Reuter, in the 1960s discovered Lactobacillus reuteri species; which was known previously as "Lactobacillus ferment biotype II", a lot of research work has been triggered to isolate the culture from different sources, study the factors affecting their growth and conduct animal and human trails to evaluate they health impact as a powerful probiotic candidate. The organism was not only isolated from human intestinal and feces but also from many mammals and vertebrates. In human trails, $L$. reuteri were consistently isolated from same subjects over experimental period thus supported that the microbe is constitute stable component of the human microbiome [1].

Lactobacillus reuteri DSM 12246 has been found capable to produce high amount of reuterin; a broad spectrum antimicrobial substance derived from glycerol, able to cease the growth of different pathogens in meat and dairy products [2] [3]. Recently, reuterin production was optimized in semi-hard cheese using L. reuterias adjunct culture [4]. Genotypic characteristics are becoming an indispensable figure of probiotic candidates in human to assign the probiotic effects to well-identified strains hence, avoid label misleading and maintain consumer confidence [5]. Recently, the defining of potential effects of probiotics on the intestinal microbiota, nutrition, immunity and mechanism of action are depending on genomic analysis of new target probiotics [6]. Selection criteria of probiotic strains include survival during transit and colonization of the human gastrointestinal tract [7].

The present study aimed to characterize the molecular and probiotic features of $L$. reuteri DSM 12246 further, investigate the effect of $\mathrm{pH}$ changes on fermentation parameters included biomass production and metabolic profile in batchculture.

\section{Materials and Methods}

\section{Microorganism, medium and inoculum preparation}

Lactobacillus reuteri DSM 12246, previously known as L. reueti 12002 [8] was stored at $-80^{\circ} \mathrm{C}$. For excremental trials, the culture was propagated in modified MRS (m-MRS) as previously described [8] for 16 hours at $37^{\circ} \mathrm{C}$.

\section{Sequencing of $16 \mathrm{~S}$ rRNA gene}

The culture of L. reuteri DSM 12246 were subjected to molecular characterization by sequencing of 16SrRNA gene at AAT-Advanced Analytical Technologies srl., Piacenza, Italy. The culture was cultivated an aerobically (Anaerogen ${ }^{\mathrm{TM}}$; Oxoid, Basingstoke, Hampshire, UK) on MRS agar for 16 hours. To extract DNA, pure colonies were treated by Fast Prep-24 (MP Biomedicals, UK) inaccordance to manufacturer instructions. DNA was amplified using p10f, p1100r, p765f and p782r primers pair complementary to the conserved regions of $16 \mathrm{~S}$ rRNA gene and PCR amplicons were used as templates for sequencing of the whole 16S rRNA gene (BMR Bio Molecular Research, Padova University, Italy). Six sequences of $16 \mathrm{~S}$ rRNA gene of L. reuteri DSM 12246 strain were obtained. 
Sequence results from each amplified fragment were aligned and assembled into contigs to obtain of the entire 1500-bp consensus sequence. Consensus sequence was compared with available sequences in GenBank using the BLASTN tool through the National Centre for Biotechnology Information (NCBI) server (https://blast.ncbi.nlm.nih.gov/Blast.cgi).

\section{RAPD-PCR analysis}

DNA was extracted from pure colonies using FTA Starter pack (What man, Maidstone, UK) in accordance with manufacturer instruction. RAPD-PCR amplification using primer RAPD2 (5'-AGCAGCGTCG-3') was performed according to Fontana et al. [9] and the amplification product was first electrophoresed at $100 \mathrm{~V}$ on $2.5 \%$ agarosegel and then stained with ethidium bromide.

\section{Determination of acid resistance}

Fresh pellets of the strain L. reuteri DSM 12246 grown in MRS broth for 16 hours were centrifuged, washed with PBS buffer (50 mmol, $\mathrm{pH}$ 7). The cells resuspended at concentration of $\mathrm{OD}_{600}=1$ inacid solution containing pepsin $\mathrm{A}$ (1\%) adjusted at $\mathrm{pH} 3$ with $\mathrm{HCl} 1 \mathrm{M}$ for 1 hour at $37^{\circ} \mathrm{C}$. The viable counts were determined before and after 60 minutes of acid treatment by plating on MRS agar (Oxoid) after serial 10-fold dilution in maximum recovery diluent. Plates were incubated an aerobically at $37^{\circ} \mathrm{C}$ for 48 hours.

\section{Resistance to simulated gastric and pancreatic juices}

To determine the upper gastro intest in altransit tolerance of $L$. reuteri DSM 12246 , a 16 hours culture was separated by centrifugation, washed and resuspended in PBS ( $\mathrm{pH}$ 7.0), then were divided into two aliquots before inoculated in simulated gastric and pancreatic juices as described by Charteris et al. [10]. Bacterial suspension was inoculated at $37^{\circ} \mathrm{C}$ in a fresh daily prepared simulated solution of gastric juice containing pepsin $(0.3 \% ; \mathrm{NaCl} 0.5 \%)$ from porcinestomach mucosa (Sigma-Aldrich) at $\mathrm{pH} 2.0$ and in pancreatic intestinal juice $(\mathrm{pH} 8)$ containing pancreatin USP $(0.1 \% ; \mathrm{NaCl} 0.5 \%)$. To determine the survival rate, aliquots were taken directly after inoculation and intervals of 1,90 and $180 \mathrm{mi}-$ nutes for gastric juice treatment and after 1 and 240 minutes in case of pancreatin juice. The viability was assessed by plating on MRS agar (Oxoid). Plates were incubated an aerobically at $37^{\circ} \mathrm{C}$ for 48 hours.

\section{Resistance to bile salt}

Bile tolerance of $L$. reuteri DSM 12246 was evaluated according to Kaushik et al. [11] using Ox bile commercially available preparation (Fluka, Sigma-Aldrich; cat \#70168). Fresh culture of L. reuteri DSM 12246 strain was inoculated in MRS broth (Oxoid) supplemented with $0.3 \%$ and $0.5 \%(\mathrm{w} / \mathrm{v})$ of bile salts followed by incubation at $37^{\circ} \mathrm{C}$. Aliquots were withdrawn at 0,1 and 2 hours intervals thenplated on MRS agar (Oxoid) and incubated an aerobically at $37^{\circ} \mathrm{C}$ for 24 hours.

\section{Adhesion to $\mathrm{CaCO}_{2}$ cells}

The adhesion to $\mathrm{CaCO}_{2}$ cells test was performed in outsourcing service at $\mathrm{Pa}$ dova University (Italy). The ability of $L$. reuteri DSM 12246 to adhere to intestinal mucosa was determined on human $\mathrm{CaCO}_{2}$ cells an intestinal epithelial cell line. Vital bacteria were labeled with fluorescent probe 5 -carboxyfluorescein di- 
acetate, Acetoxymethyl ester (5-CFDA, AM, Molecular Probes, Invitrogen) and $\cong 10^{8} \mathrm{cfu} / \mathrm{ml}$ of culture were added to cell monolayers (MOI 1:10) in absence of antibiotics. Cells and bacteria were co-incubated 120 minutes at $37^{\circ} \mathrm{C}$ and then non adhering bacteria were removed by washing three times in sterile PBS buffer. Cells were then treated with PBS-Triton $0.5 \%$. Adhering bacteria were quantified by measuring the fluorescence at $485 \mathrm{~nm}$ excitation and $535 \mathrm{~nm}$ emissions, using a fluorescence plate reader (Infinite 200, Tecan). Assays were performed in duplicate three times. One-way ANOVA test followed by a Bonferroni's multi-comparison test was performed to determine statistical significance.

\section{Antibiotic susceptibility profile}

Strain sensitivity to antibiotics was evaluated by means of serial two-fold dilution procedures in agar as suggested by the EFSA Scientific Opinion [12]. Minimum inhibitory concentration (MICs) of nine antibiotics was determined for the strain according to the ISO 10932 IDF 223:2010 [13]. After incubating the plates under anaerobic conditions at $37^{\circ} \mathrm{C}$ for 48 hours MIC values were read as the lowest concentration of an antimicrobial agent that inhibits bacterial visible growth. The test was performed as two replicates. The accuracy of susceptibility testing was monitored by parallel use of the quality control strain L. paracasei ATCC 334 which should use as internal standard according to the ISO 10932 protocol.

Effect of $\mathrm{pH}$ on biomass and metabolic profile in batch-culture

The culture was grown in modified MRS substrate (m-MRS) (sodium acetate and Tween 80 concentrations of $1.5 \mathrm{~g} / \mathrm{l}$ and $1.2 \mathrm{~g} / \mathrm{l}$, respectively. Glucose was separately sterilized and added to the broth to give a final concentration of $1 \%$ $(\mathrm{w} / \mathrm{v})$.

Batch cultures experiments were carried out in Applikon fermenter connected with Applikon ADI 1020 control unit (Applikon ${ }^{\bowtie}$ Biotechnology B.V., Delft, Netherlands). The fermenter conditions were set as $900 \mathrm{ml}$ effective fermenter volume, $250 \mathrm{rpm}$ stirring rate, continuous flushing with pure sterilized nitrogen (99.998\% purity) to maintain anaerobic conditions and the temperature was set at $37^{\circ} \mathrm{C}$. The effect $\mathrm{pH}$ was done in $\mathrm{m}-\mathrm{MRS}$ broth at $\mathrm{pH}$ rang of $3.7,4.3,5.0,5.5$, 6.0 and 6.7 which maintained by the addition of $1.5 \mathrm{~mol} \mathrm{NaOH}$ or $1.5 \mathrm{~mol}$ $\mathrm{H}_{3} \mathrm{PO}_{4}$. Each batch was run for 12 hours and samples were withdrew interval of 2 hours [8].

\section{Measurement of biomass concentration and growth rate}

The growth was followed by measuring optical density at $600 \mathrm{~nm}\left(\mathrm{OD}_{600}\right)$ using a Shimadzu UV-1201 spectrophotometer (Shimadzu Co., Japan). Samples were diluted appropriately to allow the $\mathrm{OD}_{600}$ to fall between values of 0.05 and 0.5. Cell dry weight (CDW) was calculated on the basis of measured optical density using a previously prepared standard curve of plotting $\mathrm{OD}_{600}$ values $v \boldsymbol{s}$ $\mathrm{CDW}$. The specific growth rate was calculated by plotting $\mathrm{OD}_{600} v s$. time.

\section{Measurement of substrate components and metabolites}

Glucose, acetate, lactate, and ethanol were measured using an HP1100 HPLC 
(Agilent, USA). Collected samples were centrifuged to separate the pellet then filtered using $0.2 \mu$ disc filters. Separation proceeded on a HPX-87H column $\left(300 \times 7.8 \mathrm{~mm}, 9 \mu\right.$ particle size, Bio-Rad, Richmond, CA)at $30^{\circ} \mathrm{C}$ with a cation H1 Micro-Guard column $(30 \times 4.6 \mathrm{~mm}$, Bio-Rad $)$ at an eluent flow-rate of 0.6 $\mathrm{ml} / \mathrm{min}$ of $10 \mathrm{mmol} / 1 \mathrm{H}_{2} \mathrm{SO}_{4}$. Metabolites were identified and quantified using an HP1047A refractive index detector in compared to retention times with those of standards (Sigma) [8].

\section{Results and Discussion}

\section{Molecular characterization}

Figure 1 presents the partial nucleotide sequence (length $=1565$ ) of $16 \mathrm{~S}$ rRNA of $L$. reuteri DSM 12246. Sequence similarity analyses were done using Blast searches for L. reuteri DSM 12246 against sequences available in gene bank. Phylogenetic tree construction multiple sequence alignments performed using ClustalWv1.81 with default based on $16 \mathrm{~S}$ rRNA parameters. On the basis of the result multiple alignments a phylogenetic tree was calculated for each strain by applying the using the Neighbor-joining method implanted in MEGA software version 4.1.

Species identification of the strain was determined on the basis of the highest scores obtained by the alignment with known species. For strain DSM 12246 the highest similarity score was obtained with L. reuteri strain I49 KR 36477 (100\%) and ATCC 5573016S ribosomal RNA gene (99\% of identity). Meanwhile, L. reutei DSM 12246 showed lower similarity when compared to other Lactobacillus species; i.e. L. sharpeae NR044711, L. rhamnosus AB008211.1 and L. casei NR041893.1 (Figure 2).

RAPD patterns obtained for L.reuteri DSM 12246 is shown in Figure 3. Depend on used primer the L. reuteri DSM 12246 exhibited six amplified DNA fragments within size range between 240 to $600 \mathrm{bp}$. The RAPD primer was not directed against a particular genetic locus, this resulted in band patterns often exhibit a poor reproducibility. For this reason, based on accumulating evidence from previous studies, PFGE is considered best for strain molecular typing because of its sensitivity, consistency and accuracy.

NGTTTGATCCTGGCTCAGGATGAACGCCGGCGGTGTGCCTAATACATGCAAGTCGTACGCACTGGCCCAACTGATTGATGGTGC TTGCACCTGATTGACGATGGATCACCAGTGAGTGGCGGACGGGTGAGTAACACGTAGGTAACCTGCCCCGGAGCGGGGGATAA CATTTGGAAACAGATGCTAATACCGCATAACAACAAAAGCCACATGGCTTTTGTTTGAAAGATGGCTTTGGCTATCACTCTGGG ATGGACCTGCGGTGCATTAGCTAGTTGGTAAGGTAACGGCTTACCAAGGCGATGATGCATAGCCGAGTTGAGAGACTGATCGG CCACAATGGAACTGAGACACGGTCCATACTCCTACGGGAGGCAGCAGTAGGGAATCTTCCACAATGGGCGCAAGCCTGATGGA CCACAATGGAACTGAGACACGGTCCATACTCCTACGGGAGGCAGCAGTAGGGAATCTTCCACAATGGGCGCAAGCCTGATGGA GCAACACCGCGTGAGTGAAGAAGGGTTTCGGCTCGTAAAGCTCTGTTGTTGGAGAAGAACGTGCGTGAGAGTAACTGTTCACG CAGTGACGGTATCCAACCAGAAAGTCACGGCTAACTACGTGCCAGCAGCCGCGGTAATACGTAGGTGGCAAGCGTTATCCGGA TTTATTGGGCGTAAAGCGAGCGCAGGCGGTTGCTTAGGTCTGATGTGAAAGCCTTCGGCTTAACCGAAGAAGTGCATCGGAAAC CGGGCGACTTGAGTGCAGAAGAGGACAGTGGAACTCCATGTGTAGCGGTGGAATGCGTAGATATATGGAAGAACACCAGTGGC GAGGCGGCTGTCTGGTCTGCAACTGACGCTGAGGCTCGAAAGCATGGGTAGCGAACAGGATTAGATACCCTGGTGGCAGT CGACCGCAAGGTTGAAACTCAAAGGAATTGACGGCGCCCTTCAGTGCCGGAGCTAACGCATAATCACTCGAAGCTACGCGAA GAACCTTACCAGGTCTTGACATCTTGCGCTAACCTTAGAGATAAGGCGTTCCCTTCGGGGACGCAATGACAGGTGGTGCATGGT CGTCGTCAGCTCGTGTCGTGAGATGTTGGGTTAAGTCCCGCAACGAGCGCAACCCTTGTTACTAGTTGCCAGCATTAAGTTGGG COTCGTCAGC TCGT CACTCTAGTAGACTGCCGTGACAAACCGGAGGAAGGTGGGGACGACGTCAGATCATCATGCCCCTTATGACCTGGGCTACA CACGTGCTACAATGGACGGTACAACGAGTCGCAAGCTCGCGAGAGTAAGCTAATCTC TAAAGCCGTCTCAGTTGGACTGTA GGCTGCAACTCGCCTACACGAAGTCGGAATCGCTAGTAATCGCGGATCAGCATGCCGCGGTOAATACGTTCCCGGCCTIGTAC ACACCGCCCGTCACACCATGGGAGTTTGTAACGCCCAAAGTCGGTGGCCTAACCATTATGGAGGGAGCCGCCTAAGGCGGGAC AGATGACTGGGGTGAAGTCGTAACAAGGTAGCCGTAGGAGAACCTGCGGCTGGATCACCTCCA

Figure 1. Nucleotide sequence of 16S rRNA of L. reuteri DSM 12246. 


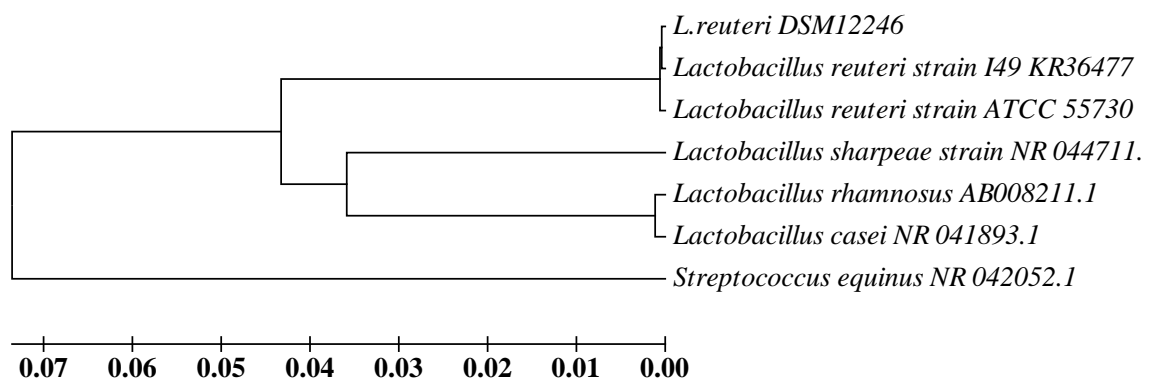

Figure 2. Phylogenetic tree analysis of L. reuteri DSM 12246 and the lineage of related species based on $16 \mathrm{~S}$ rRNA nucleotide sequences.

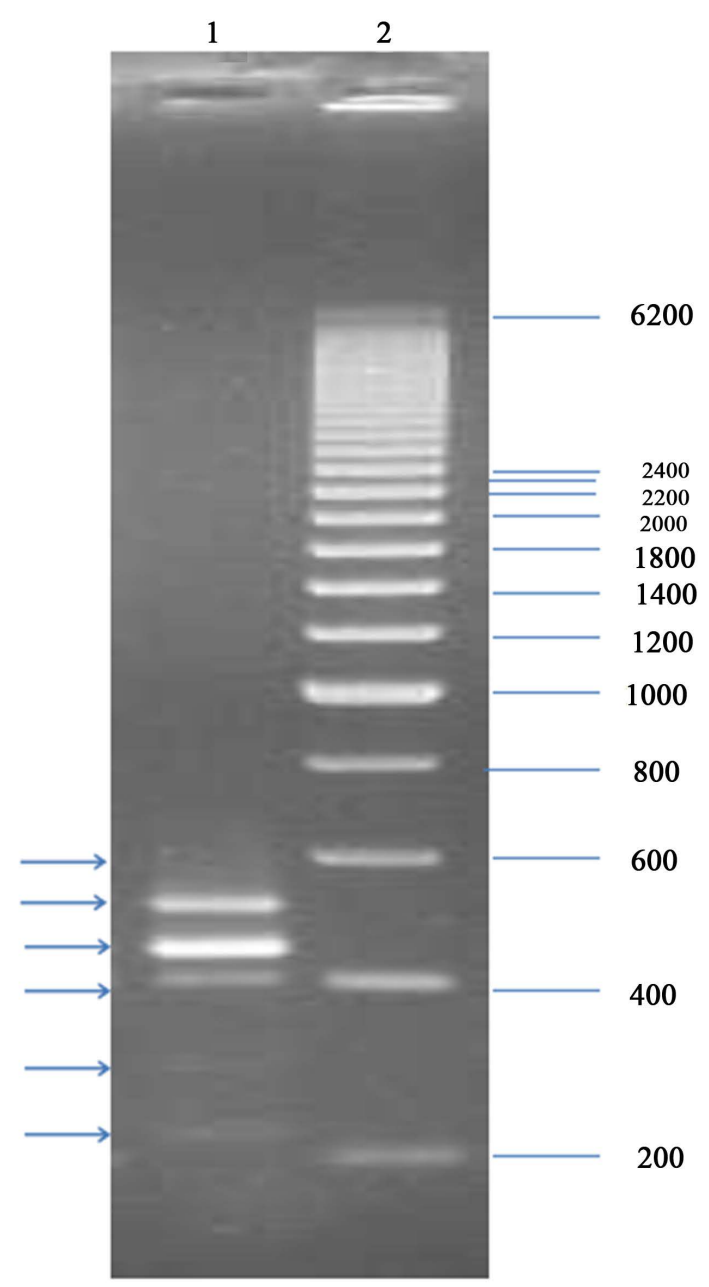

Figure 3. RAPD profile of L. reuteri DSM 12246 (lane 1) in compared to molecular weight marker 200 bp (Promega, Italy) (lane 2).

\section{Probiotic characteristics}

\section{Resistance to gastrointestinal barriers}

The effect of different gastrointestinal barriers against $L$. reuteri DSM 12246 is shown in Table 1 . The viability of $L$. reuteri DSM 12246 was reduced by $0.7 \log _{10}$ $\mathrm{cfu} / \mathrm{ml}$ after one hour of incubation in acid juice. Exposed of L. reuteri DSM 12246 to simulated gastric juice caused a loss of viability by $3 \log _{10}$ after three 
Table 1. Probiotic characteristics and resistance to gastrointestinal biological barriers of $L$. reuteri DSM 12246.

\begin{tabular}{ccl}
\hline Treatments & Time (min) & CFU/ml \\
\hline Acid juice & 0 & $6.3 \times 10^{8}$ \\
& 60 & $1.1 \times 10^{8}$ \\
Simulated gastric juice & 0 & $2.0 \times 10^{8}$ \\
& 1 & $2.0 \times 10^{8}$ \\
& 90 & $6.5 \times 10^{7}$ \\
& 180 & $1.6 \times 10^{5}$ \\
& 0 & $2.0 \times 10^{8}$ \\
& 1 & $1.4 \times 10^{8}$ \\
& 240 & $1.3 \times 10^{8}$ \\
$0.3 \%$ of bile salts & 0 & $7.5 \times 10^{6}$ \\
& 60 & $7.0 \times 10^{6}$ \\
& 120 & $6.7 \times 10^{6}$ \\
& 00 & $6.0 \times 10^{6}$ \\
& 120 & $6.0 \times 10^{6}$ \\
& & $0.3 \times 10^{6}$ \\
\hline
\end{tabular}

hours while, stimulated pancreatic juice did not demonstrate significant inhibitory effect as the viable count only decreased by $0.1 \log _{10} \mathrm{cfu} / \mathrm{ml}$. Similarly, $L$. reuteri DSM 12246 at concentration of $0.3 \%$ and $0.5 \%$ Ox bile salts were resistible only decreased by 0.05 and $0.1 \log _{10} \mathrm{cfu} / \mathrm{ml}$ respectively.

\section{Adhesion to $\mathrm{CaCO}_{2}$ cells}

The absolute number of adherent bacterial cells to $\mathrm{CaCO}_{2}$ an intestinal epithelial cell line is presented in Figure 4. L. reuteri DSM 12246 showed better adhesion ability to human intestinal epithelial cells, as opposed to the negligible adhesion of $L$. paracasei and $L$. gasseri, although resulted significantly less efficient than L. rhamnosus.

\section{Antibiotic susceptibility profile}

Out of nine types of tested antibiotics, L. reuteri DSM 12246 showed to be sensitive to eight of it with equal or lower values than cut-off limits established by EFSA for L. reuteri (Table 2). In general, the reduced susceptibility of lactobacilli to aminoglycosides is thought to occur because of membrane impermeability [14]. It has also been documented that the presence of MRS medium may interfere with the susceptibility testing of specifically the aminoglycosides [15]. The MIC values for erythromycin were above to the EFSA cut-off for L. reuteri species (Table 2). L. reuteri DSM 12246 strain grew to higher dilutions of antibiotic tested for erythromycin $(8 \mu \mathrm{g} / \mathrm{ml})$. 


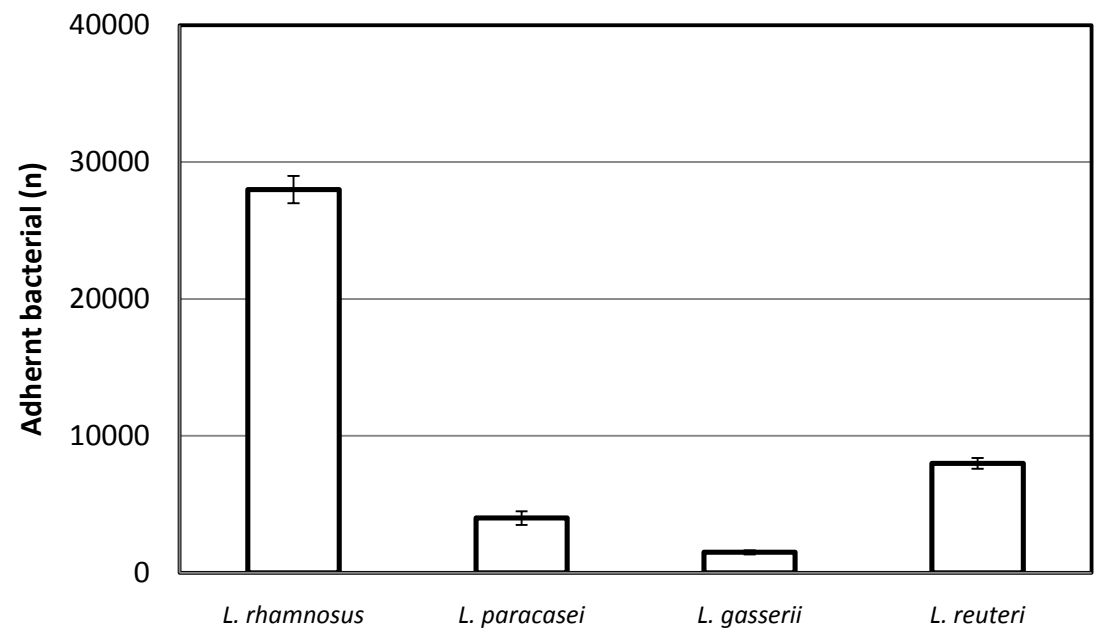

Figure 4. Number of adherent bacterial cells of L. reuteri DSM 12246 to $\mathrm{CaCO}_{2}$ cells.

Table 2. Antibiotic susceptibility (MICs value, $\mu \mathrm{g} / \mathrm{ml}$ ) of L. reuteri DSM 12246 in agar dilution method.

\begin{tabular}{ccc}
\hline Antibiotic & L. reuteri DSM $\mathbf{1 2 2 4 6}$ & ${ }^{a}$ EFSA breakpoint valueof $L$. reuteri \\
\hline Gentamycin & 4 & 8 \\
Kanamycin & 64 & 64 \\
Streptomycin & 32 & 64 \\
Tetracycline & 8 & 16 \\
Erythromycin & 8 & 1 \\
Clindamycin & 4 & 4 \\
Chloramphenicol & 4 & 4 \\
Ampicillin & 1 & 2 \\
Neomycin & 8 & 8
\end{tabular}

${ }^{a}$ Adapted from Branton et al. [17].

Resistance to erythromycin is commonly attributed to erm genes. In a previous study Egervärn and collaborators [16] reported genetic characterization of 38 strains of $L$. reuteri from various human and animal habitats revealed that tet (W) and erm genes were found in almost all $L$. reuteri strains with atypical MICs for tetracycline and/or erythromycin and thus appear to be the most common resistance determinants within this species. Seven genes were found to be associated with antibiotic resistance, including a beta-lactamase, a MATE family multiantimicrobial extrusion protein, and 2 topoisomerase and 2 DNA gyrase genes associated with resistance to fluoroquinolones. These antibiotic resistance elements were demonstrated to be common to other lactobacilli [17]. The transferability of the tetracycline resistance gene tet $(\mathrm{W})$ of $L$. reuteri as a donor of resistance genes in the human gut to related lactobacilli and unrelated genus of enterococci, and bifidobacteria was investigated meanwhile, no gene transfer was demonstrated under the tested conditions [18]. 
In most lactobacilli antibiotic genetic codes carried on plasmids hence, plasmid curing technique could be used to avoid any possibility of gene transformation. Curing plasmids $(\mathrm{n}=2)$ from L. reuteri ATCC 55730 by protoplast technique resulted in a daughter antibiotic sensitive strain, DSM 17938 which is used in clinical trials [19]. The FDA evaluation considered L. reuteri strain DSM 17938 as GRAS [20].

\section{Effect of $\mathrm{pH}$ on biomass and metabolites profile in batch culture}

Glucose converted by heterofermentative lactic acid bacteria to produce equimolecular amounts of lactate, acetate and carbon dioxide or ethanol [21]. Changes in the culture conditions might lead in the prevalence of one of these metabolites. When L. reuteri was cultured in m-MRS maintained at $\mathrm{pH}$ rang of $3.7,4.3,4.7,5.0,5.5$ and 6.7 in batch-culture results, several differences in the growth rate and metabolites were observed (Figure 5 and Figure 6 and Table 3 ). The specific growth rate reached its maximum at $\mathrm{pH} 5.5(\mu=0.641 / \mathrm{h})$ and reduced at lower and higher $\mathrm{pH}$ values. Similarly, biomass followed the same pattern (Figure 5).

Figures 6(a)-(f) presented the kinetics of both substrate consumption and end-productformation at different $\mathrm{pH}$ values. At low $\mathrm{pH}$ values of 4.3 and 4.7,

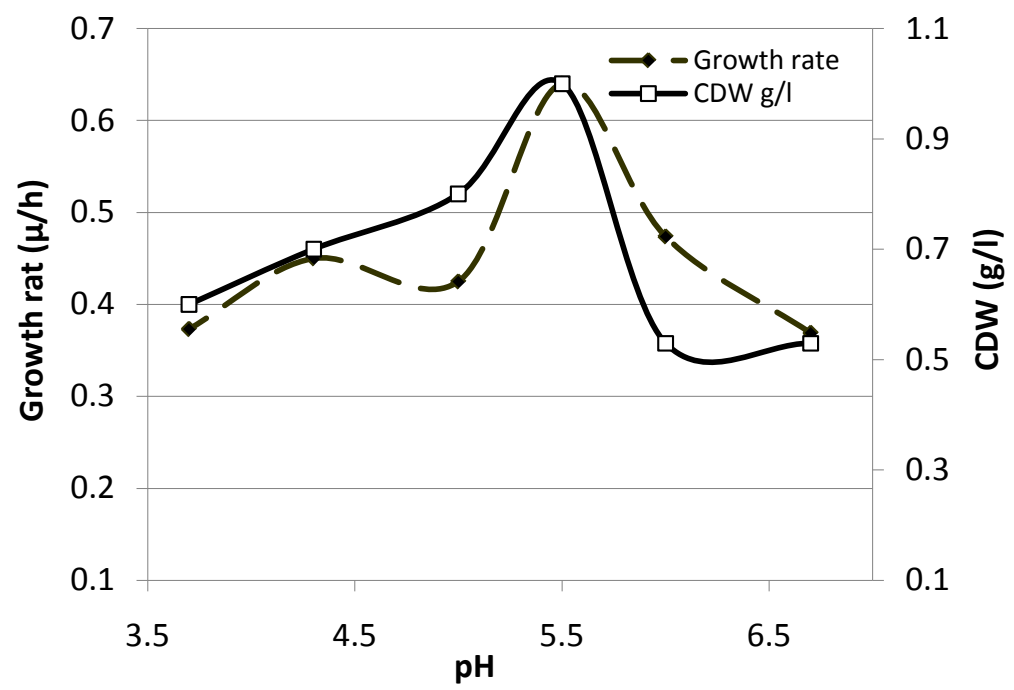

Figure 5. The specific growth rate and cell dry weight of $L$ reuteriDSM 12246 during $12 \mathrm{~h}$ incubation in batch-culture at different $\mathrm{pH}$ values. - - growth rate; - - cell dry weight.

Table 3. Effect of $\mathrm{pH}$ on lactate, acetate and ethanol production in batch-culture of $L$. reuteri DSM 12246 grown under anaerobic conditions.

\begin{tabular}{ccccccc}
\hline \multirow{2}{*}{ Yields } & \multicolumn{6}{c}{$\mathrm{pH}$ values } \\
\cline { 2 - 7 } & 3.7 & 4.3 & 5.0 & 5.5 & 6.0 & 6.7 \\
\hline$Y_{\text {lactate }}$ & 29.7 & 25.0 & 45.0 & 49.0 & 55.5 & 68.5 \\
$Y_{\text {acetate }}$ & 3.1 & 4.3 & 4.6 & 5.9 & 7.1 & 4.8 \\
$Y_{\text {ethanol }}$ & 25.9 & 29.0 & 59.0 & 62.5 & 61.5 & 83.9 \\
\hline
\end{tabular}




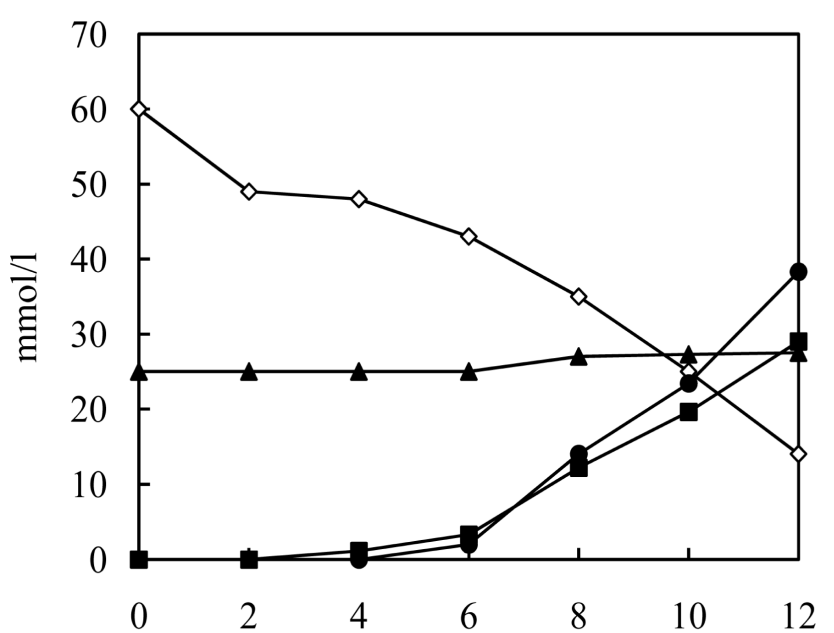

(a)

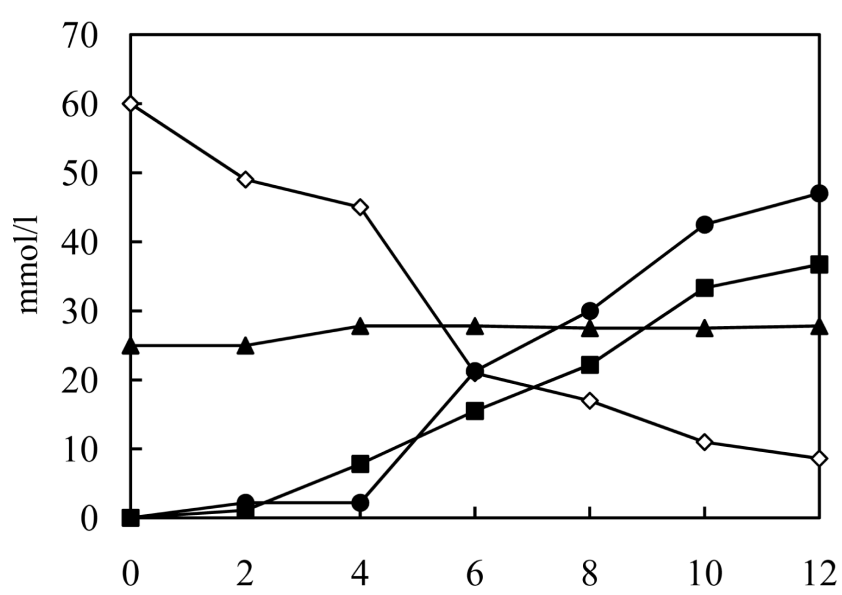

(c)

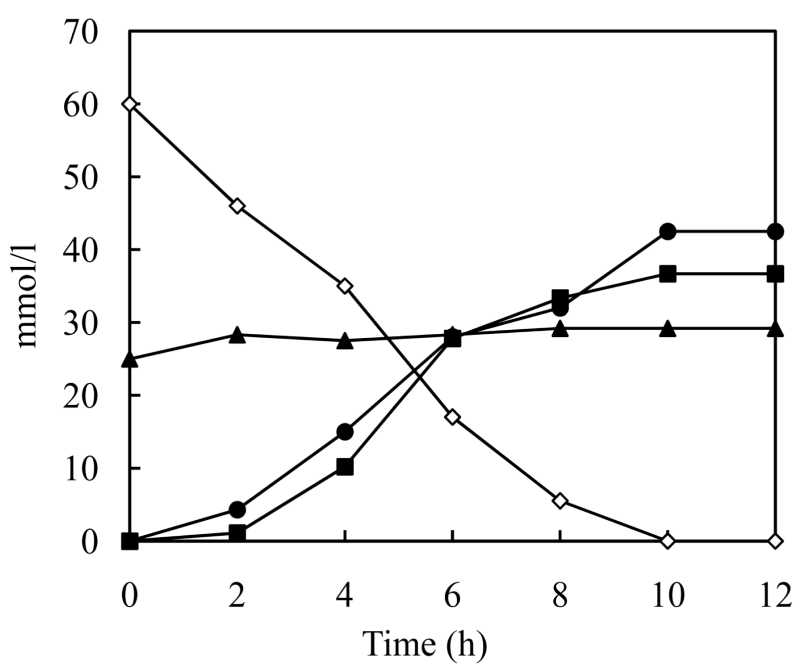

$\diamond$ glucose $\rightarrow$ - lactate $\multimap$ - acetate $\multimap$ - ethanol

(e)

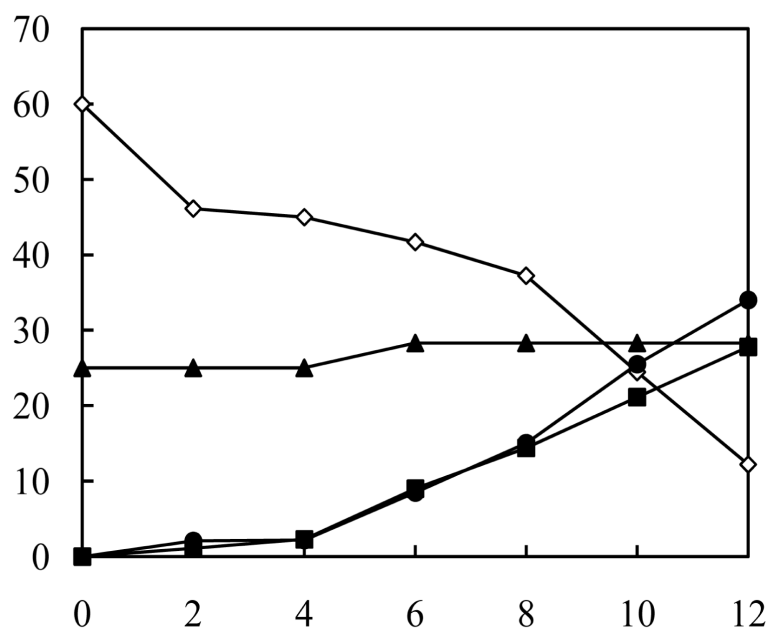

(b)

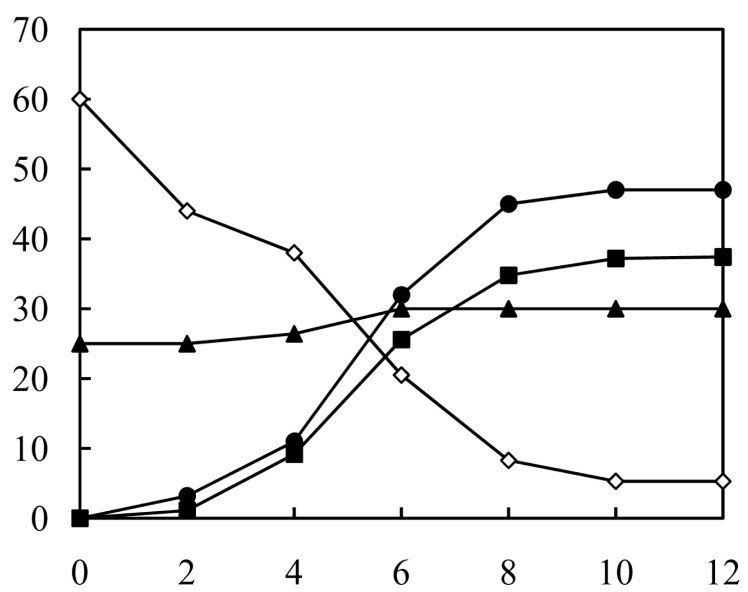

(d)

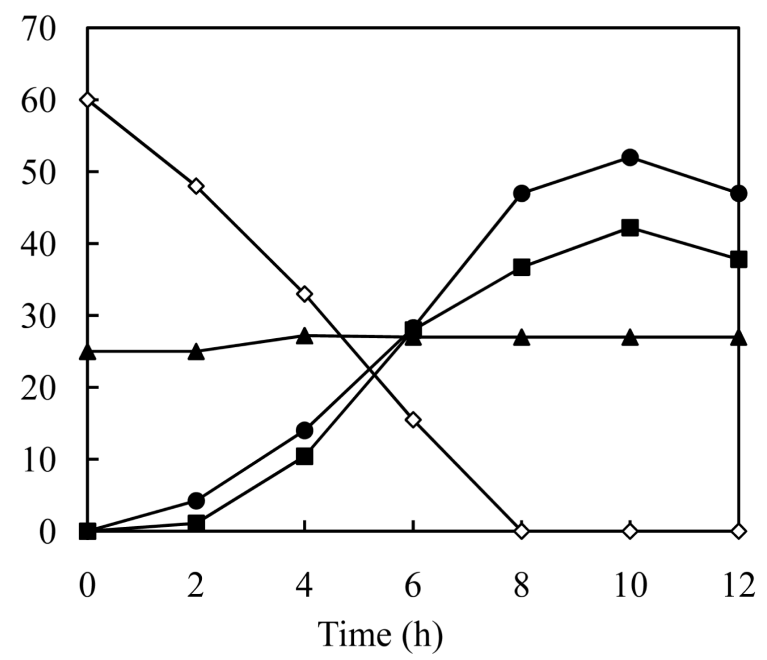

$\diamond$ glucose $\rightarrow$ - lactate $\multimap$ acetate $\multimap$ ethanol

(f)

Figure 6. Effect of $\mathrm{pH}$ on metabolic profile by L. reuteri DSM 12246 in batch-culture. (a) $\mathrm{pH} 3.7$; (b) $\mathrm{pH} 4.3$; (c) $\mathrm{pH} 5.0$; (d) $\mathrm{pH}$ 5.5; (e) $\mathrm{pH} 6.0$; (f) $\mathrm{pH}$ 6.7. $\multimap-$ residual glucose;

lactate; $\multimap$ acetate; $\multimap$ ethanol. 
glucose utilization was low but kept efficient, 46 and $48 \mathrm{mmol}$ residual sugar was found respectively at the end of incubation (Figure 6(a) and Figure 6(b)). Format was not detected in any of the fermentations. Increasing $\mathrm{pH}$ to6.7 coincided with fast sugar depletion ( 8 hours) and the highest rate of glucoseutilization (Figure 6(f)). It is reported that heterofermentative lactobacilli (i.e. L. buchneri and $L$. brevis) cannot grow under anaerobic conditions on glucose, because they cannot reduce acetyl phosphate to ethanol, an essential product to maintain the overall redox balance [21]. In contradiction the behavior of $L$. reuteri DSM 12246 was quite different as ethanol was the major end-product at all $\mathrm{pH}$ values tested under anaerobic conditions. In agreement with present results, the same behavior of $L$. reuteri was reported by Ragout et al. [22].

At $\mathrm{pH} 4.3$, culture began to produce lactate 2 hours earlier than ethanol at glucose depletion percent of $20 \%$ and $28 \%$ for each metabolite respectively (Figure 6(a)). Meanwhile, ethanol became a dominate metabolite after 12 hours. Acetate profile appeared not changed, a small increasing concentration being found after 8 hours and kept at the same level until the end of incubation time. A comparable trend was noticed at $\mathrm{pH} 4.7$ with a typical plateaus were observed of both substrate consumption and metabolites formation (Figure 6(b)).

Ethanol production rate speeded up as $\mathrm{pH}$ culture increased (Figure 6(c) and Figure 6(f)). Meanwhile at the end of incubation, no significant change of final concentration was observed at pH's 5.0 to 6.7. In parallel with glucose depletion, the concentration of ethanol was 1.2 times that of the acids (Figure 6(e) and Figure 6(f)). A similar pattern of events was reported for other microorganisms [23]. It is suggested that the switch in metabolites from organic acids to ethanol synthesis might provide some protection of injured cells as a result of overacidification in the growth medium [24].

Shifting the $\mathrm{pH}$ value from 3.7 to 6.7 led to increase the yield of ethanol per cell biomass $\left(Y_{x / p}\right)$ by 3.24 -foldafter 12 hours of fermentation (Table 3). The $Y_{\text {lactate }}$ also showed a significant increase from $Y_{p H 3.7}=29.7$ to $Y_{p H 6.7}=68.5 \mathrm{mmol} / \mathrm{g}$. The $Y_{\text {acetate }}$ remained fairly constant; $\cong 3.1-4.6 \mathrm{mmol} / \mathrm{g}$ at lower $\mathrm{pH}$ values while only increased at pH's 5.5 and 6.0 by 1.9 and 2.29 -folds respectively (Table 3 ). This is might be due to acetate consumption by $L$. reuteri which previously produced it [22]. Form the present results it is postulated that $L$. reuteri DSM 12246 not only possess the ability to tolerate acid conditions but also to produce high amount of lactic acid in compared with other strains belong to same species [22]. These possibilities might provide the ability of the organism to dominate the growth in the surrounding environment, i.e. gastrointestinal. From the technological point of view, the ability of $L$. reuteri DSM 12246 to switch between metabolites during fermentation are of great importance since the development of flavour and texture profile during the production of dairy and sour dough are relay on the ratio between volatile and non-volatile compounds.

\section{Conclusion}

In addition to its high ability to produce antimicrobial reuterin, the present 
study concludes that L. reuteri DSM 12246 is well molecular defined and characterized probiotic able to transit and colonize in GIT, fairly grow over a range of $\mathrm{pH}$ values while it preferred acidic environment and capable to switch between metabolites in order to not only provide cell protection but give the possibility to develop flavour and texture profile in fermented products and dominate the microbial community.

\section{References}

[1] Britton, R.A. (2017) Lactobacillus reuteri. In: Floch, M.H., Ringel, Y. and Walker, W.A., Eds., The Microbiota in Gastrointestinal Pathophysiology: Implications for Human Health, Prebiotics, Probiotics, and Dysbiosis, Elsevier Inc., Amsterdam, 89-97. https://doi.org/10.1016/B978-0-12-804024-9.00008-2

[2] El-Ziney, M.G. and Debevere, J.M. (1998) The Effect of Reuterin on Listeria monocytogenes and Escherichia coli O157:H7 in Milk and Cottage Cheese. Journal of Food Protection, 10, 1275-1280. https://doi.org/10.4315/0362-028X-61.10.1275

[3] El-Ziney, M.G., van den Tempel, T., Debevere, J., et al. (1999) Application of Reuterin Produced by Lactobacillus reuteri 12002 for Meat Decontamination and Preservation. Journal of Food Protection, 62, 257-261. https://doi.org/10.4315/0362-028X-62.3.257

[4] Martin-Cabrejas, I., Langa, S., Gaya, P., et al. (2017) Optimization of Reuterin Production in Cheese by Lactobacillus reuteri. Journal of Food Science and Technology, 54, 1346-1349. https://doi.org/10.1007/s13197-017-2563-2

[5] Donelli, G., Vuotto, C. and Mastromarino, P. (2013) Phenotyping and Genotyping are Both Essential to Identify and Classify a Probiotic Microorganism. Microbial Ecology Health and Disease, 24, 20105. https://doi.org/10.3402/mehd.v24i0.20105

[6] Bongaerts, G. and Severijnen, R. (2007) Probiotics: Are They Incredible Panaceas? On the Science behind Beneficial Non-Pathogenic Microbial Agents. International Journal of Probiotics and Prebiotics, 1, 87-96.

[7] Goldin, B.R., Gorbach, S.L., Saxelin, M., et al. (1992) Survival of Lactobacillus Species (strain LGG) in Human Gastrointestinal Tract. Digestive Diseases and Sciences, 37, 121-128. https://doi.org/10.1007/BF01308354

[8] El-Ziney, M.G., Arneborg, N., Uyttendaele, M., et al. (1998) Characterization of Growth and Metabolite Production of Lactobacillus reuteri during Glucose/Glycerol Cofermentation in Batch and continuous Cultures. Biotechnology Letters, 20, 913-916. https://doi.org/10.1023/A:1005434316757

[9] Fontana, C., Sandro, C.P. and Vignolo, G. (2005) Monitoring the Bacterial Population Dynamics during Fermentation of Artisanal Argentinean Sausages. International Journal Food Microbiology, 103, 131-142. https://doi.org/10.1016/j.ijfoodmicro.2004.11.046

[10] Charteris, W.P., Kelly, P.M., Morelli, L., et al. (1998) Development and Application of an In Vitro Methodology to Determine the Transit Tolerance of Potentially Probiotic Lactobacillus and Bifidobacterium Species in the Upper Human Gastrointestinal Tract. Journal Applied Microbiology, 84, 759-768. https://doi.org/10.1046/j.1365-2672.1998.00407.x

[11] Kaushik, J.K., Kumar, A., Duary, R.K., et al. (2009) Functional and Probiotic Attributes of an Indigenous Isolate of Lactobacillus plantarum. PLoS One, 4. https://doi.org/10.1371/journal.pone.0008099 
[12] EFSA Scientific Opinion (2012) Guidance on the Assessment of Bacterial Susceptibility to Antimicrobials of Human and Veterinary Importance. European Safety Authority Journal, 10, 2740.

[13] ISO 10932 | IDF 223:2010 (2010) Milk and Milk Products-Determination of the Minimal Inhibitory Concentration (MIC) of Antibiotics Applicable to Bifidobacteria and Non-Enterococcal Lactic Acid Bacteria (LAB).

[14] Elkins, C.A. and Mullis, L.B. (2004) Bile-Mediated Aminoglycoside Sensitivity in Lactobacillus Species Likely Results from Increased Membrane Permeability Attributable to Cholic Acid. Applied and Environmental Microbiology, 12, 7200-7209. https://doi.org/10.1128/AEM.70.12.7200-7209.2004

[15] Huys, G., D’Haene, K. and Swings, J. (2002) Influence of the Culture Medium on Antibiotic Susceptibility Testing of Food-Associated Lactic Acid Bacteria with the Agar Overlay Disc Diffusion Method. Letters in Applied Microbiology, 34, 402-406. https://doi.org/10.1046/j.1472-765X.2002.01109.x

[16] Egervärn, M., Roos, S. and Lindmark, H. (2009) Identification and Characterization of Antibiotic Resistance Genes in Lactobacillus reuteri and Lactobacillus plantarum. Journal of Applied Microbiology, 107, 1658-1668. https://doi.org/10.1111/j.1365-2672.2009.04352.x

[17] Branton, W.B., Jones, M.L., Tomaro-Duchesneau, C., et al. (2011) In Vitro Characterization and Safety of the Probiotic Strain Lactobacillus reuteri Cardioviva NCIMB 30242. International Journal of Probiotics Prebiotics, 6, 1-12.

[18] Egervärn, M., Lindmark, H., Olsson, H., et al. (2010) Transferability of a Tetracycline Resistance Gene from Probiotic Lactobacillus reuteri to Bacteria in the Gastrointestinal Tract of Humans. Antonie van Leeuwenhoek, 97, 189-200. https://doi.org/10.1007/s10482-009-9401-0

[19] Rosander, A., Connolly, E. and Roos, S. (2008) Removal of Antibiotic Resistance Gene-Carrying Plasmids from Lactobacillus reuteri ATCC 55730 and Characterization of the Resulting Daughter Strain, L. reuteri DSM 17938. Applied and Environmental Microbiology, 74, 6032-6040. https://doi.org/10.1128/AEM.00991-08

[20] FDA (2008). https://wayback.archive-it.org/7993/20170606195210/https://www.fda.gov/Food/In gredientsPackagingLabeling/GRAS/NoticeInventory/ucm154990.htm

[21] Kandler, O. (1983) Carbohydrate Metabolism in Lactic Acid Bacteria. Antonie van Leeuwenhoek, 49, 209-224. https://doi.org/10.1007/BF00399499

[22] Ragout, A., Siñeriz, F., Diekmann, H., et al. (1994) Effect of Environmental pH on the Fermentation Balance of Lactobacillus reuteri. Journal of Applied Bacteriology, 77, 388-391. https://doi.org/10.1111/j.1365-2672.1994.tb03439.x

[23] Dainty, R.H. and Hofman, F.J.K. (1983) The Influence of Glucoseconcentration and Culture Incubation Time on End-Product Formation during Aerobic Growth of Brochothrix thermosphacta. Journal of Applied Bacteriology, 55, 233-239. https://doi.org/10.1111/j.1365-2672.1983.tb01320.x

[24] Speck, E.L. and Freese, E. (1973) Control of Metabolite Secretionin Bacillus subtilis. Journal of General Microbiology, 78, 261-275.

https://doi.org/10.1099/00221287-78-2-261 